\title{
The ASY-EOS experiment at GSI: Constraining the symmetry energy at supra-saturation densities
}

P. Russotto ${ }^{1}$, M. Chartier ${ }^{2}$, M.D. Cozma $^{3}$, E. De Filippo ${ }^{1}$,

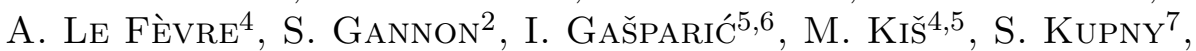

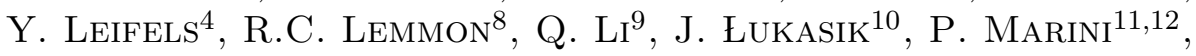

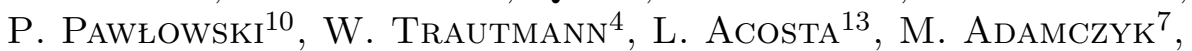
A. Al-AJlan ${ }^{14}$, M. Al-Garawi ${ }^{15}$, S. Al-Homaidhi ${ }^{14}$, F. Amorini ${ }^{13}$,

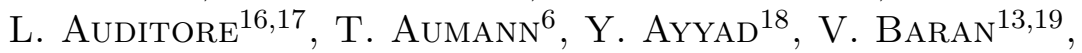
Z. BAsrak ${ }^{5}$, R. BAssini ${ }^{20}$, J. Benlliure ${ }^{18}$, C. Boiano ${ }^{20}$, M. Boisjoli ${ }^{12}$, K. Boretzky ${ }^{4}$, J. BrzyChCZYK ${ }^{7}$, A. Budzanowski ${ }^{10}$, G. Cardella ${ }^{1}$, P. Cammarata ${ }^{21}$, Z. Chajecki ${ }^{22}$, A. Chbihi $^{12}$, M. Colonna ${ }^{13}$, B. Czech $^{10}$, M. Di Toro ${ }^{13,23}$, M. Famiano ${ }^{24}$, V. Greco ${ }^{13,23}$, L. Grassi ${ }^{5}$, C. Guazzoni ${ }^{20,25}$, P. Guazzoni ${ }^{20,26}$,

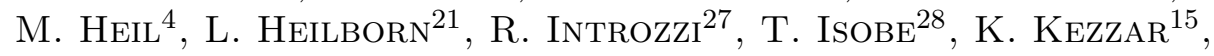
A. Krasznahorkay ${ }^{29}, \mathrm{~N} . \mathrm{KurZ}^{4}$, E. La Guidara ${ }^{1}$,

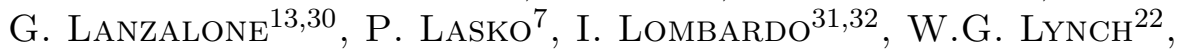
Z. Matthews ${ }^{2}$, L. MaY ${ }^{21}$, T. Minniti $^{1}$, M. Mostazo ${ }^{18}$, A. Pagano $^{1}$, M. Papa ${ }^{1}$, S. Pirrone ${ }^{1}$, R. PleskaC ${ }^{4}$, G. Politi ${ }^{1,23}$, F. Porto ${ }^{13,23}$,

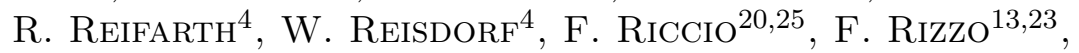

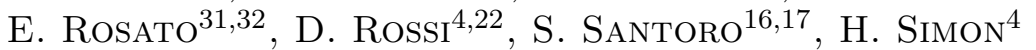

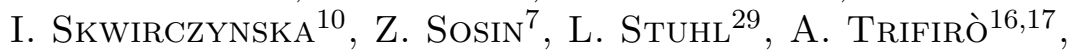
M. Trimarchi ${ }^{16,17}$, M.B. Tsang ${ }^{22}$, G. $\operatorname{Verde}^{1}$, M. Veselsky ${ }^{33}$,

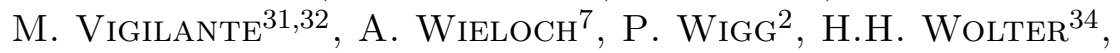

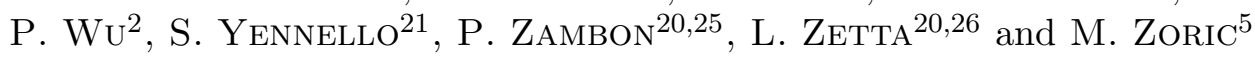


${ }^{1}$ INFN, Sezione di Catania, Catania, Italy

${ }^{2}$ University of Liverpool, Liverpool, UK

3 IFIN-HH, Măgurele-Bucharest, Romania

${ }^{4}$ GSI Helmholtzzentrum, Darmstadt, Germany

5 Ruder Boškovic̀ Institute, Zagreb, Croatia

6 Technische Universität, Darmstadt, Germany

7 Jagiellonian University, Kraków, Poland

8 STFC Laboratory, Daresbury, UK

${ }^{9}$ Huzhou Teachers College, China

10 IFJ-PAN, Kraków, Poland

11 CENBGn Univ. de Bordeaux, CNRS/IN2P3, 33175 Gradignan, France

12 GANIL, Caen, France

13 INFN-Laboratori Nazionali del Sud, Catania, Italy

14 KACST Riyadh, Riyadh, Saudi Arabia

15 King Saud University, Riyadh, Saudi Arabia

16 INFN, Gruppo Collegato di Messina, Messina, Italy

17 Università di Messina, Messina, Italy

18 University of Santiago de Compostela, Santiago de Compostela, Spain

19 University of Bucharest, Bucharest, Romania

${ }^{20}$ INFN, Sezione di Milano, Milano, Italy

${ }^{21}$ Texas A\&M University, College Station, TX, USA

22 NSCL Michigan State University, East Lansing, MI, USA

23 Università di Catania, Catania, Italy

24 Western Michigan University, Kalamazoo, MI, USA

25 Politecnico di Milano, Milano, Italy

26 Università di Milano, Milano, Italy

27 INFN, Politecnico di Torino, Torino, Italy

28 RIKEN, Wako, Japan

29 Institute of Nuclear Research, Debrecen, Hungary

30 Università Kore, Enna, Italy

31 INFN, Sezione di Napoli, Napoli, Italy

32 Università di Napoli, Napoli, Italy

33 Institute of Physics, Slovak Academy of Sciences, Bratislava, Slovakia 34 LMU, München, Germany 


\begin{abstract}
The elliptic-flow ratio of neutrons with respect to protons or light complex particles in reactions of heavy ions at pre-relativistic energies has been proposed as an observable sensitive to the strength of the symmetry term of the nuclear equation of state at supra-saturation densities. In the ASY-EOS experiment at the GSI laboratory, flows of neutrons and light charged particles were measured for ${ }^{197} \mathrm{Au}+{ }^{197} \mathrm{Au}$, ${ }^{96} \mathrm{Zr}+{ }^{96} \mathrm{Zr}$ and ${ }^{96} \mathrm{Ru}+{ }^{96} \mathrm{Ru}$ collisions at $400 \mathrm{MeV} /$ nucleon with the Large Area Neutron Detector LAND as part of a setup with several additional detection systems used for the event characterization. Flow results obtained for the $\mathrm{Au}+\mathrm{Au}$ system, in comparison with predictions of the UrQMD transport model, confirm the moderately soft to linear density dependence of the symmetry energy deduced from the earlier FOPI-LAND data.
\end{abstract}

\title{
1 Introduction
}

The neutron-proton elliptic flow ratio and difference have been proposed as effective probes of the high-density behavior of the nuclear symmetry energy [1-3]. The comparison of existing data from the FOPI-LAND experiment [4] with calculations performed with the UrQMD transport model [5] suggests a moderately soft to linear symmetry term. The result suffers, however, from the considerable statistical uncertainty of the experimental data $[1,3]$. The same data set was also compared to calculations performed with the Tübingen QMD model and a constraint compatible with the UrQMD result was obtained [6]. At the same time, a thorough study of the parameter dependence of the model predictions was performed in order to devise a route towards a model independent constraint of the the high-density symmetry energy [6]. It was, in particular, found that different parameterizations of the isovector part of the equation of state, the Gogny inspired (momentum dependent) vs. the power law (momentum independent) potential, lead to very similar results.

In order to improve the statistical accuracy of the experimental flow parameters for the $\mathrm{Au}+\mathrm{Au}$ reaction and to extend the flow measurements to other systems, the symmetric collision systems ${ }^{197} \mathrm{Au}+{ }^{197} \mathrm{Au},{ }^{96} \mathrm{Zr}+{ }^{96} \mathrm{Zr}$ and ${ }^{96} \mathrm{Ru}+{ }^{96} \mathrm{Ru}$ at $400 \mathrm{MeV} /$ nucleon incident energies have been chosen for the ASY-EOS experiment, conducted at the GSI laboratory in May 2011 (S394 experiment). As in the previous experiment, the Large Area Neutron Detector LAND [7] was used for the detection and identification of neutrons and light charged particles. By including the KRATTA telescope array [8] in the setup, the study of isospin effects in these reactions was extended to 
additional observables as, e.g., the yield ratios of light isobar pairs ${ }^{3} \mathrm{H} /{ }^{3} \mathrm{He}$ and ${ }^{7} \mathrm{Li} /{ }^{7} \mathrm{Be}$.

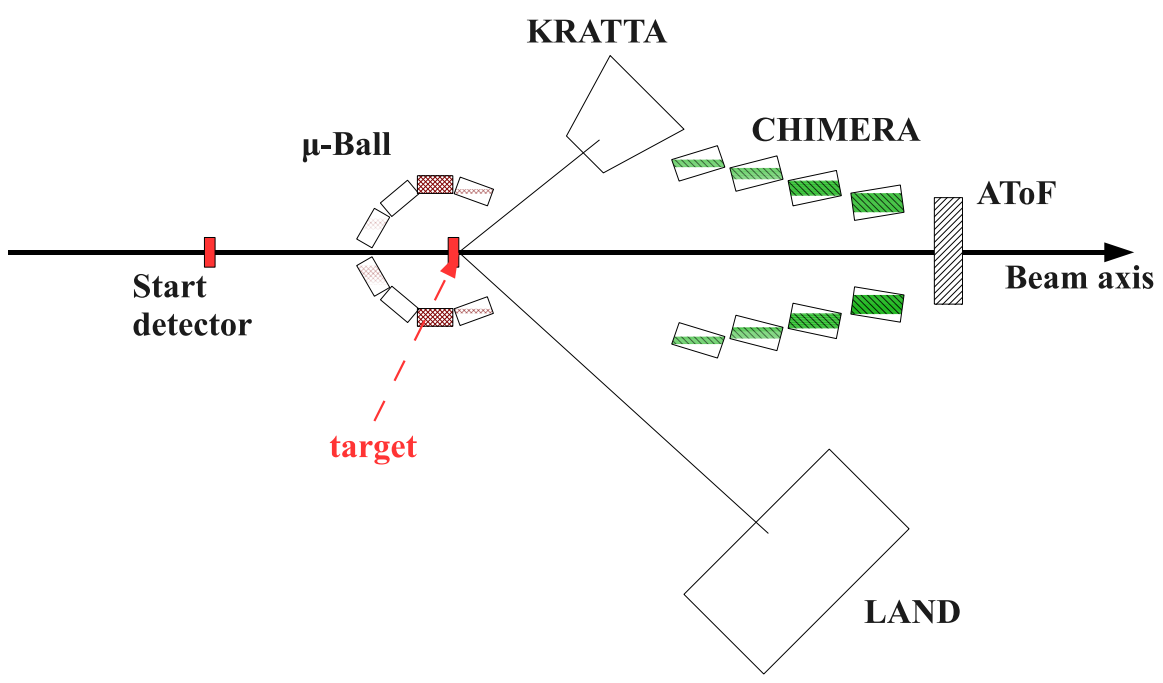

Figure 1: Schematic view of the experimental setup of the ASY-EOS experiment S394 at GSI (not to scale).

\section{ASY-EOS experiment at GSI}

A schematic view of the experimental set-up is shown in Fig. 1. The Large Area Neutron Detector (LAND) [7] was positioned to cover laboratory angles around $45^{\circ}$ with respect to the beam direction, at a distance of about $5 \mathrm{~m}$ from the target. A veto-wall of plastic scintillators in front of LAND allowed discriminating neutrons and charged particles. In this configuration, it was possible to measure the direct and elliptic flows of neutrons and charged particles at mid-rapidity with high precision in the same angular acceptance. In addition, the Krakòw Triple Telescope Array, KRATTA [8], was installed to measure the energy, emission angles and isotopic composition of light charged reaction products. The 35 modules of KRATTA were arranged in a $7 \times 5$ array and placed opposite to LAND at a distance of $40 \mathrm{~cm}$ from the target. They covered $160 \mathrm{msr}$ of solid angle at polar angles between $20^{\circ}$ and $64^{\circ}$. The modules of KRATTA consisted of two, optically decoupled, $\mathrm{CsI}(\mathrm{Tl})$ crystals (thickness of 2.5 and $12.5 \mathrm{~cm}$ ) and three large area, $500 \mu \mathrm{m}$ thick, PIN photo-diodes. Very good isotopic resolution has been obtained in the whole dynamic range up to $Z \sim 6$ [9]. 
The determination of the impact parameter and the orientation of the reaction plane required the use of several devices: i) the ALADIN Time-ofFlight (AToF) wall [10] was used to detect charged particles at polar angles smaller than $7^{\circ}$; two walls (front and rear) of $2.5^{*} 100 \mathrm{~cm}^{2}$ plastic scintillators, read by two photo-multipliers at both ends, gave information on emission angle, atomic number and velocity of forward-emitted ions; ii) 50 thin (between 3.6 and $5.6 \mathrm{~mm}$ ) $\mathrm{CsI}(\mathrm{Tl})$ elements, read out by photo-diodes and arranged in 4 rings, of the Washington-University $\mu$ Ball array [11], surrounded the target at polar angles between $60^{\circ}$ and $147^{\circ}$ with the aim of measuring the multiplicty and angular distribution of backward emitted particles in order to discriminate against background reactions on non-target material; iii) $352 \mathrm{CsI}(\mathrm{Tl})$ scintillators, $12 \mathrm{~cm}$ thick, of the CHIMERA multidetector [12], arranged in 8 rings with $2 \pi$ azimuthal coverage around the beam axis and covering polar angles between $7^{\circ}$ and $20^{\circ}$, were used to detect light charged particles from mid-rapidity emissions.

With beam intensities of about $10^{5}$ pps and targets of $1-2 \%$ interaction probability, about $5 * 10^{6}$ events were collected for each system. Special runs were performed with and without target, in order to measure the background from interaction of projectile ions with air, and with iron shadow bars in front of LAND or the neutron background measurement.

\section{Data analysis}

For rejecting background reactions due to the interaction of Au projectiles with non-target material (mainly air), the correlation of the two reaction plane orientations given by CHIMERA and by the $\mu$ Ball detectors, was used, as shown in fig. 2. From the $\mu$ Ball data, the reaction plane orientation has been estimated by averaging over the azimuthal directions of the recorded hits, i.e. by calculating a $\vec{Q}$ vector (see Ref. [13] for definition) as $\vec{Q}=$ $\sum_{i=1}^{N} \hat{p}_{t}^{i}$, where $\hat{p}_{t}^{i}$ is the transverse momentum vector (with respect to the beam direction) of the $i^{t h}$ hit and $N \geq 2$. For CHIMERA, the $\vec{Q}$ vector has been calculated as $\vec{Q}=\sum_{i=1}^{N} Z^{i} \beta_{t}^{i} \gamma^{i}$, with $N \geq 4$, taking only the forward emitted particles with rapidity in the center of mass reference frame $y_{i}^{C M}>0.1$.

Figure 2 shows the correlation between CHIMERA and $\mu$ Ball reaction plane orientations for $\mathrm{Au}+\mathrm{Au}$ reactions (panel a)) and $\mathrm{Au}+$ Empty Frame (panel b)) data, relative to each other according to the integrated beam intensity. In panel a), the strong anti-correlation for on-target reactions is evident, as expected because CHIMERA covers the forward and the $\mu$ Ball 

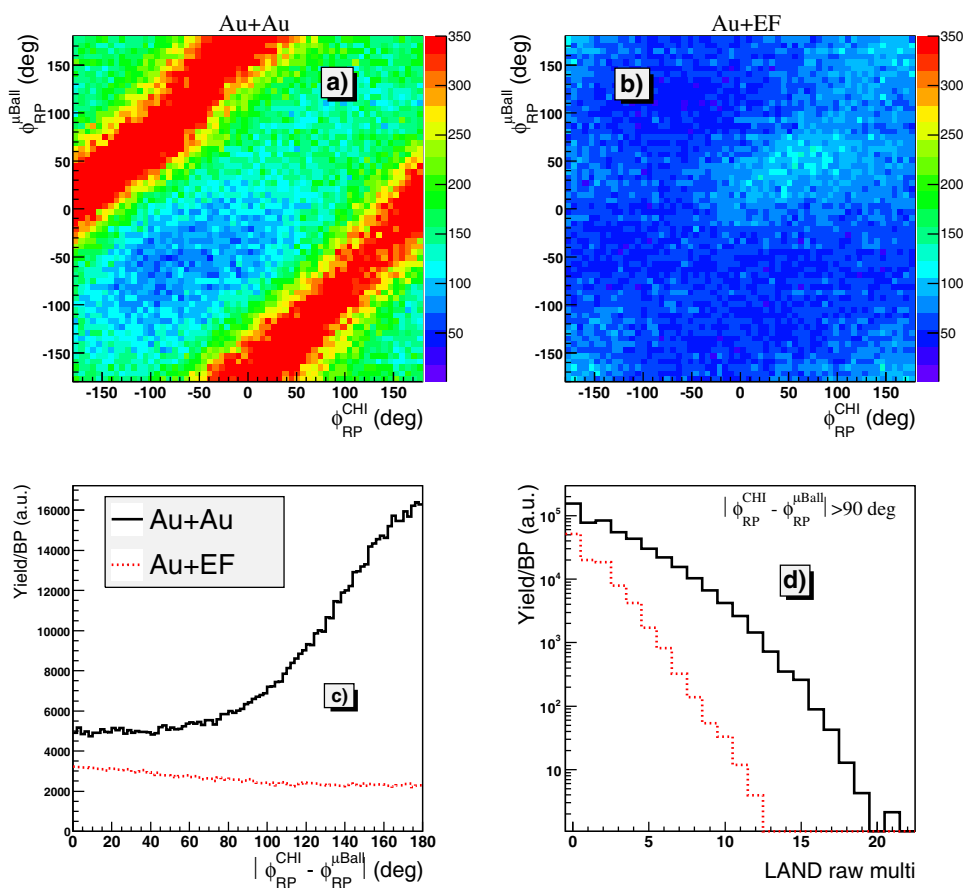

Figure 2: Correlation between the two reaction plane orientations given by CHIMERA ( $\mathrm{x}$ axis) and by the $\mu$ Ball (y axis) for $\mathrm{Au}+\mathrm{Au}$ (panel a) and $\mathrm{Au}+$ no target $(\mathrm{EF}=$ Empty Frame, panel $\mathrm{b})$ data; difference of the two reaction plane orientations for $\mathrm{Au}+\mathrm{Au}$ and for $\mathrm{Au}+$ no target data (panel c)), normalized with respect to the integrated beam intensity, and the raw multiplicity registered with LAND for $\mathrm{Au}+\mathrm{Au}$ (full line) and for $\mathrm{Au}+$ no target data (dotted, panel d).

mainly the backward hemispheres in the c.m. frame. In runs with empty target frames, the recorded yields are low and only a weak positive correlation is observed. The difference between the two reaction plane orientations, normalized with respect to the integrated beam intensity, is presented in panel c). In order to minimize the contributions of non-target collisions in the data analysis, an anti-correlation of the CHIMERA and $\mu$ Ball reactionplane orientations was required. The condition $\left|\Phi_{R P}^{C H I}-\Phi_{R P}^{\mu B a l l}\right|>90^{\circ}$ was applied, resulting in a relative weight of background reactions of less than $20 \%$. This clearly shows the relevance of the $\mu$ Ball data in rejecting background reactions.

Panel d) of Fig. 2 shows the LAND raw multiplicity (number of fired paddles) normalized with respect to the integrated beam intensity for $\mathrm{Au}+\mathrm{Au}$ and $\mathrm{Au}+$ no target data, after applying the indicated CHIMERA- $\mu$ Ball an- 

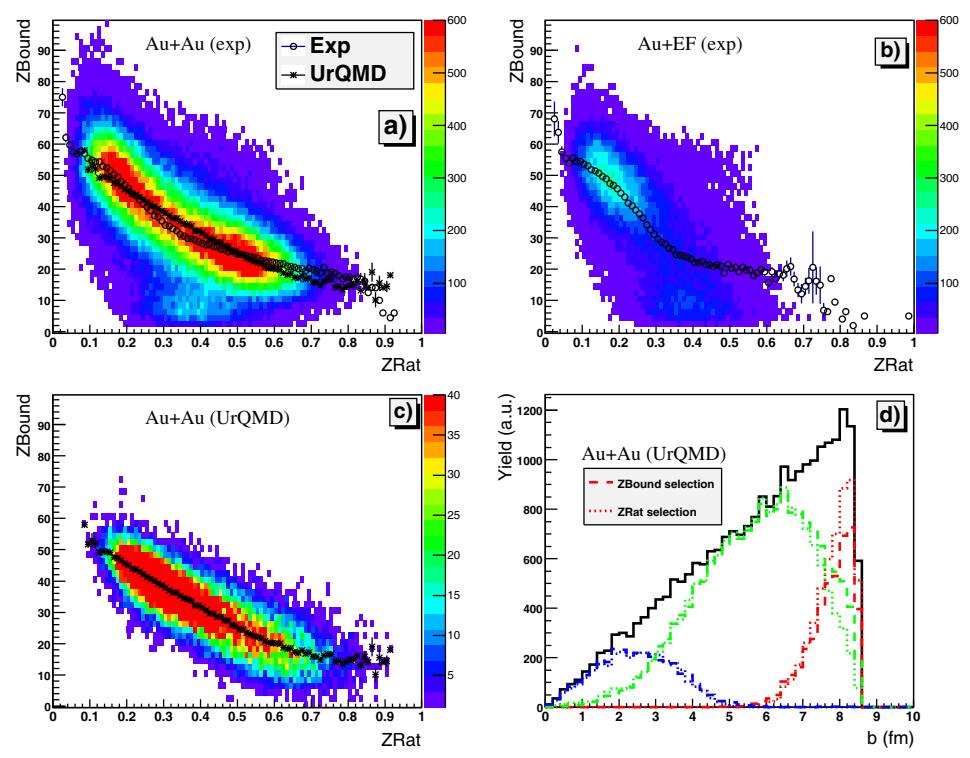

Figure 3: (Colour online) panel a): ZBound versus ZRat correlation plot for $\mathrm{Au}+\mathrm{Au}$ at $400 \mathrm{MeV} /$ nucleon; panel b): same as panel a) for $\mathrm{Au}+$ no target (EF) collisions; panel c): ZBound versus ZRat correlation plot from UrQMD calculations for $\mathrm{Au}+\mathrm{Au}$ at $400 \mathrm{MeV} /$ nucleon and impact parameter $\mathrm{b}<8.5 \mathrm{fm}$; panel d) UrQMD simulated impact parameter distribution (black solid line). Blue, green and red lines show impact parameter distributions when selecting $\mathrm{b}<3 \mathrm{fm}, 3<\mathrm{b}<7.5 \mathrm{fm}$, b>7.5 fm, by gating on ZBound (dashed) and ZRat (dotted).

ticorrelation condition. The contribution from non-target backgrounds in the kinematic region of LAND is apparently rather weak.

For the impact parameter selection global variables were constructed from the CHIMERA and AToF data. They included $Z$ Bound $=\sum_{i=1}^{N} Z^{i}$ with $Z^{i} \geq 2$ and the ratio of transverse to longitudinal charge, $Z$ Rat $=$ $10 * Z_{\text {Trans }} / Z_{\text {Long }}$, where $Z_{\text {Trans }}=\sum_{i=1}^{N} Z_{i} * \sin ^{2}\left(\theta_{i}\right), Z_{\text {Long }}=\sum_{i=1}^{N} Z_{i} *$ $\cos ^{2}\left(\theta_{i}\right)$ and $\theta_{i}$ is the polar angle of the $i^{t h}$ particle in the laboratory reference system. The choice of such variables as impact parameter selectors has been guided by UrQMD calculations for given impact parameter ranges, filtered for angular acceptance, detection thresholds and resolution of the detectors.

The ZBound vs. ZRat correlation plot for $\mathrm{Au}+\mathrm{Au}$ reactions is presented in panel a) of Fig. 3. A strong anti-correlation is found between the two global variables, the smaller ZBound, the larger ZRat and, according to UrQMD calculations, the smaller the impact parameter. The same correlation for $\mathrm{Au}+$ no target data is shown in panel b) and the corresponding correlation predicted by the UrQMD model for $\mathrm{Au}+\mathrm{Au}$ reactions with im- 


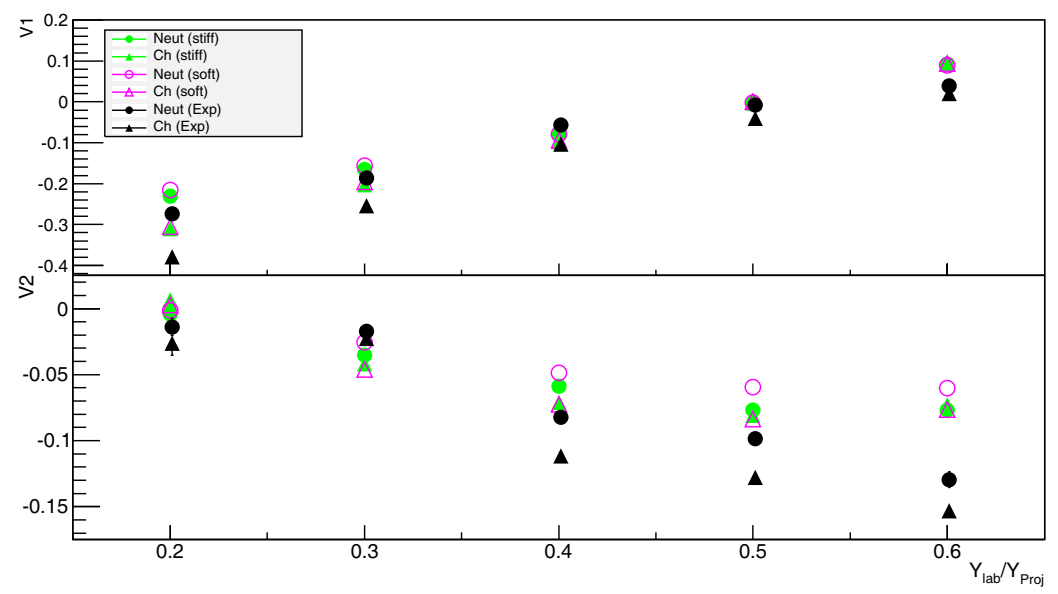

Figure 4: (Colour online) Measured flow parameters v1 (top) and v2 (bottom) for $\mathrm{b}<7.5 \mathrm{fm} \mathrm{Au}+\mathrm{Au}$ collisions at $400 \mathrm{MeV} /$ nucleon for neutrons (black full dots) and charged particles (black full triangles) as a function of the normalized rapidity $Y_{l a b} / Y_{\text {proj }}$. The UrQMD predictions for neutrons and charged particles obtained with a stiff ( $\gamma=1.5$, green full symbols) and a soft $(\gamma=0.5$, purple empty symbols) density dependence of the symmetry term have been filtered to correspond to the geometrical acceptance of the experiment. The experimental data have been corrected for the dispersion of the reaction plane.

pact parameter $b<8.5 \mathrm{fm}$ is presented in panel c). There is good qualitative agreement between the experimental data and the calculations. In panel a) the measured and the calculated $<$ ZBound $>$ vs. ZRat correlations are shown by full and open black triangles, respectively. The impact parameter intervals obtained by gating on specific ZBound and/or ZRat intervals are shown in panel d) of Fig. 3).

An important parameter for flow studies is the resolution achieved in determining the azimuthal orientation of the reaction plane. It largely determines the uncertainty associated with the determined flow parameters [14]. The resolution achieved in reconstructing the reaction plane orientation was tested using the sub-event mixing technique as described in Ref. [15]. The reaction-plane dispersion parameter $\chi$ and the corresponding correction factors for the Fourier parameters v1 and v2 characterizing the measured azimuthal distributions were estimated. The analysis performed so far shows that the actual reaction plane orientation achieved with the CHIMERA detector modules is better than previously estimated with simulations. 

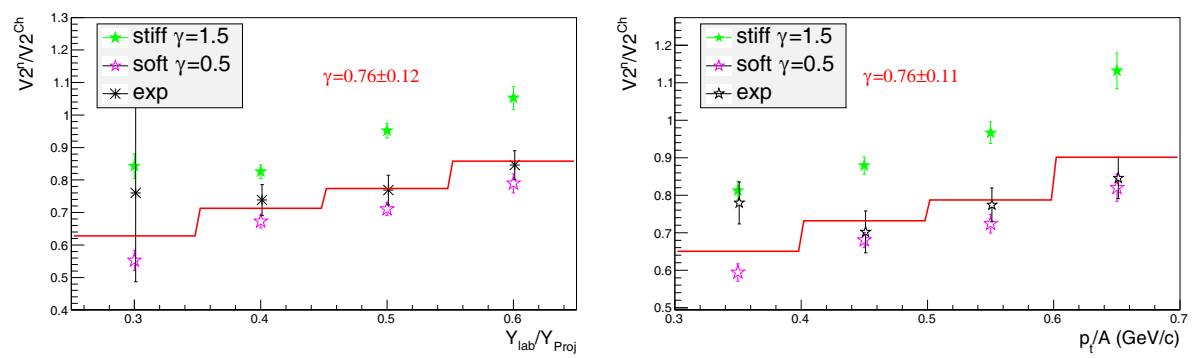

Figure 5: (Colour online) Elliptic flow ratio of neutrons and charged particles for moderately central $(b<7.5 \mathrm{fm})$ collisions of $\mathrm{Au}+\mathrm{Au}$ at $400 \mathrm{MeV} /$ nucleon, as a function of the normalized rapidity $y_{l a b} / y_{\text {proj }}$ (left panel) and of the transverse momentum per nucleon $p_{t} / A$ (right panel). The black symbols represent the experimental data. The UrQMD predictions for stiff ( $\gamma=1.5$, green symbols) and soft $(\gamma=0.5$, purple symbols $)$ are shown. The red line in each panel is the result of a linear interpolation between the predictions; the obtained gamma values and their uncertainties are indicated.

\section{Elliptic neutron and charged-particle flows}

Azimuthal distributions of neutrons and light charged particles measured with LAND with respect to the reaction plane given by CHIMERA have been extracted for $\mathrm{Au}+\mathrm{Au}$ reactions from runs with and without the shadow bar. After subtracting the background, the obtained distributions have been fitted with formula (1) of Ref. [1] in order to determine the direct (v1) and elliptic (v2) flow coefficients. Due to insufficient energy resolution in the LAND veto-wall, charge identification with the $\Delta$ E-vs.-time-of-flight technique has not been possible. Therefore, the results for neutrons and for all recorded charged particles $(\mathrm{Ch})$ are presented. The v1 and v2 parameters for $\mathrm{Ch}$ and neutrons as functions of the reduced rapidity $Y_{l a b} / Y_{\text {proj }}$ are shown in the upper and lower panels of Fig. 4, respectively. In the same figure, also the UrQMD predictions for a soft $(\gamma=0.5)$ and a stiff $(\gamma=1.5)$ symmetry energy potential [1] are given.

\section{A new constraint for the symmetry energy}

Constraints for the symmetry energy were determined as in Ref. [1] by comparing the ratios of the elliptic flows of neutrons and $\mathrm{Ch}, v 2^{n} / v 2^{C h}$, with the corresponding UrQMD predictions for the soft and stiff cases. The results obtained as functions of the reduced rapidity and of the transverse 
momentum $p_{t} / A$ are shown in Fig. 5. A preliminary value for the power-law coefficient $\gamma$, deduced by linearly interpolating between the predictions, is $\gamma=0.76 \pm 0.12$ and $\gamma=0.76 \pm 0.11$ for the rapidity and $p_{t}$ sorted data, respectively. The new constraint is compatible with the previous one presented in Ref. [1] by using FOPI-LAND data and the same UrQMD model but the statistical error has been significantly reduced by a factor of $\sim 2$. The determination of systematic uncertainties is still on-going; a preliminary analysis indicates that they are substantial and may lead to an overall final uncertainty of the order of $\Delta \gamma \approx \pm 0.25$.

\section{Outlook}

Comparisons of the experimental data with other theoretical transport models will be useful in order to pursue the route towards a model-independent constraint of the high-density symmetry energy initiated in Ref. [6]. The promising preliminary results of the present experiment may also be seen as an encouragement for extending the measurement of neutron and charged particle flows to other reaction systems and energies. Future experiments may thereby benefit from the unique possibilities offered by the NeuLAND detector presently constructed as part of the $R^{3} B$ experimental set-up [16] and from the availability of radioactive ion beams for reaction studies at FAIR.

\section{References}

[1] P. Russotto et al., Phys. Lett. B697 (2011) 471.

[2] M.D. Cozma, Phys. Lett. B700 (2011) 139.

[3] P. Russotto et al., Eur. Phys. Jour. A50 (2014) 38, and refs. therein.

[4] Y. Leifels et al., Phys. Rev. Lett. 71 (1993) 963.

[5] Q. Li et al., J. Phys G 31 (2005) 1359.

[6] M.D. Cozma et al., Phys. Rev. C88 (2013) 044912.

[7] Th. Blaich et al., NIM A314 (1992) 136.

[8] J. Lukasik et al., Nucl. Instr. and Meth. A709 (2013) 120.

[9] J. Lukasik et al., see contr. to this volume; S. Kupny et al., ibidem.

[10] A. Schüttauf et al., Nucl. Phys. A607 (1996) 457.

[11] D.G. Sarantites et al., Nucl. Instr. and Meth. 381 (1996) 418.

[12] A. Pagano et al., Nucl. Phys. A734 (2004) 504. 
[13] P. Danielewicz et al., Phys. Lett. B157 (1985) 146.

[14] A. Andronic et al., Eur. Phys. J. A30 (2008) 31.

[15] J.-Y. Ollitrault, preprint nucl-ex/971003.

[16] https://www.gsi.de/r3b 